\title{
The association between exaggeration in health related science news and academic press releases: retrospective observational study
}

This Research paper (BMJ 2014;349:g7015, doi:10.1136/bmj. g7015) contains the same typo in figures 2 and 3. In both figures, the middle title should have read: "Causal claims from correlation [not casual as was published]."
Cite this as: BMJ 2014;349:97666

๑ $\odot$ BMJ Publishing Group Ltd 2014 